\title{
VALORES HEMÁTICOS DEL RONSOCO (HYDROCHAERIS HYDROCHAERIS) EN CAUTIVERIO EN LA AMAZONÍA PERUANA
}

\author{
Karina Muñoz D. ${ }^{1}$ y Enrique Montoya G. ${ }^{2}$
}

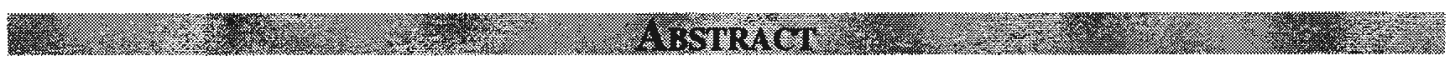

\section{Hematological values of captive bred capybara (Hydrochaeris hydrochaeris) in the Peruvian Amazon.}

\begin{abstract}
Hematological values were determined for 35 ( 19 male, 16 female, juvenile and adult) healthy, captive reared capybara (Hydrochaeris hydrochaeris) at "Biodiversidad Amazónica (BIOAM)" in Iquitos, Perú. The animals were sedated with dart delivered xilazine and ketamine, and blood samples were collected in sterile tubes containing EDTA. A Neubauer chamber was used to determine erythrocyte and leukocyte numbers, the microhematocrit method for hematocrit, the oxihemoglobin method for hemoglobin and Shilling's method for leukocyte differential counts. MCV, MCH and MCHC indices were calculated based on results of the red blood series. The following results were obtained: erythrocytes $3.19 \pm 0.157 \times 10^{6} \mathrm{ml}$, hemoglobin $14.32 \pm 0.16 \mathrm{~g} / 100 \mathrm{ml}$, hematocrit $42.23 \pm 1.52 \%$, MCV $133.53 \pm 5.92 \mathrm{fl}, \mathrm{MCH} 45.57 \pm 2.28 \mathrm{pg}, \mathrm{MCHC} 34.42$ $\pm 2.20 \mathrm{~g} / \mathrm{dl}$, leukocytes $5.40 \pm 0.31 \times 10^{3} / \mathrm{ml}$, myelocytes $0 \%$, metamyelocytes $0 \%$, band neutrophil $0.94 \pm 0.31 \%$, segmenter $51.89 \pm 3.06 \%$, eosinophils $1.57 \pm 0.59 \%$, basophiles $0 \%$, lymphocytes $42.26 \pm 3.21 \%$ and monocytes $0 \%$. No statistical differences were found between male and female. These results are similar to hematological values are similar to those reported by other authors for capybara reared in captivity, but differ from those in ranched animals.
\end{abstract}

Key words: Erythrocytes, leukocytes, capybara.

El presente estudio tuvo por objetivo determinar los valores hematológicos en ronsocos (Hydrochaeris hydrochaeris) de la Amazonía Peruana en condiciones clínicamente normales, bajo un particular sistema de manejo en cautiverio. Se emplearon ronsocos machos $(n=19)$ y hembras $(n=16)$, entre juveniles y adultos, provenientes del zoocriadero "Biodiversidad Amazónica (BIOAM)", ubicado en la ciudad de Iquitos, departamento de Loreto - Perú. Para la obtención de las muestras, los animales fueron anestesiados con xilazina en una dosis de 0.25 a $0.5 \mathrm{mg} / \mathrm{kpv}$ y ketamina en dosis de 7 - $10 \mathrm{mg} / \mathrm{kpv}$ cargados en dardos. La sangre fue colectada en tubos conteniendo EDTA (etilenodiaminotetraacético). El número de eritrocitos y leucocitos fueron determinados por el método convencional con cámara de Neubauer, el hematocrito por el método del microhematocrito, la hemoglobina por el método de la oxihemoglobina, el recuento diferencial de leucocitos utilizando la técnica de Shilling y se calcularon los índices hematemétricos (VGM, HGM y CMHG) en base a los valores hematológicos encontrados para la serie roja. El análisis estadístico se realizó utilizando la prueba de $\mathrm{T}$ de Student. Los resultados fueron para la serie eritrocítica: $3.19 \pm 0.157 \times 10^{6} \mu \mathrm{l}$; hemoglobina: $14.32 \pm 0.16 \mathrm{~g} / \mathrm{ml}$; hematocrito: $42.23 \pm 1.52 \%$; VGM: $133.53 \pm 5.92 \mathrm{fl}$;

\footnotetext{
'Práctica privada

${ }^{2}$ Estación Experimental IVITA - Iquitos - FMV - UNMSM
} 
H.G.M.: $45.57 \pm 2.28$ Pg;C.H.G.M.: $34.42 \pm 2.20 \mathrm{~g} / \mathrm{dl}$; y en la serie leucocítica: $5.40 \pm 0.31 \mathrm{x}$ $10^{3} / \mu 1$; neutrofilos en banda: $0.94 \pm 0.31 \%$; segmentados: $51.89 \pm 3.06 \%$; eosinófilos: $1.57 \pm 0.59 \%$; basófilos $0 \%$; linfocitos: $42.26 \pm 3.21 \%$. No se encontraron mielocitos, metamielocitos ni monocitos. Tampoco se encontraron diferencias estadísticas significativas entre machos y hembras.

Palabras clave: Eritrocitos, leucocitos, ronsoco.

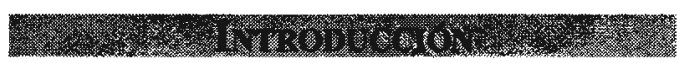

En América Latina la fauna silvestre es una importante fuente de alimento para una elevada población rural, constituyendo hasta el $85 \%$ de suministros de proteína animal. Además, la fauna silvestre proporciona cueros, pieles, fibra, guano y especies vivas, cuya venta otorga ingresos, en algunos casos elevados, a la economía local y nacional (FAO, 1988), por lo que se debe tomar medidas efectivas para una mayor protección de hábitat y especies $\mathrm{y}$, por otro lado, aplicar normas de manejo que aseguren la supervivencia, incremento y aprovechamiento racional de las especies más relevantes, particularmente aquellas como el ronsoco, que ofrece buenas perspectivas de un manejo semi intensivo o intensivo debido a la amplia posibilidad de su domesticación.

El ronsoco se puede aprovechar principalmente para la producción de carne, que se caracteriza por su excelente calidad nutricional, buen sabor, olor y textura; se puede usar en la fabricación de embutidos y conservas, según ensayos realizados en Brasil y Venezuela. El cuero es muy valioso y sirve para la confección de artículos como guantes, bolsos, etc. La crianza del ronsoco puede tener un impacto socio económico al ofrecer ocupación laboral e ingresos a quienes se dediquen a su producción (Ojasti, 1972), por lo que en la actualidad se estan realizando estudios sobre sus hábitos de reproducción y alimentación. En el campo de la Medicina Veterinaria, se hace necesario determinar los valores hematológicos normales en esta especie, a fin de interpretar las alteraciones en los diversos cuadros clínicos. El análisis de sangre es una importante ayuda, al ofrecer el conocimiento del grado y modo de respuesta del tejido hematopoyético del animal, permitiendo el diagnóstico de las afecciones hemáticas, la repercusión de las mismas sobre los elementos constituyentes de la sangre y proporciona importantes datos sobre el grado de reacción del organismo ante la presencia de algún microorganismo invasor, deficiencias nutricionales, etc.(Guerci, 1985).

El objetivo del presente trabajo fue determinar los valores hematológicos del ronsoco en cautiverio, sometido a un particular sistema de manejo y en condiciones clínicas normales, como un estudio básico que lleve al mejor conocimiento de las características de la sangre de esta especie.

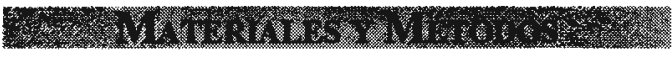

El presente estudio se realizó en animales del zoocriadero Biodiversidad Amazónica (BIOAM), ubicado en el km 23 de la Carretera Iquitos-Nauta, cerca de la ciudad de Iquitos, departamento de LoretoPerú; a una Latitud de $3^{\circ} 45^{\prime}$ Sur y Longitud de $73^{\circ} 12^{\prime}$ Oeste, temperatura promedio de $25.9^{\circ} \mathrm{C}$ y precipitación anual promedio de $2600 \mathrm{~mm}$.

Se emplearon 35 ronsocos ( 16 hembras y 19 machos). Las edades exactas de estos animales no se pudieron precisar debido a que fueron adquiridos sin tener conocimiento de ello, catalogándose solamente como juveniles y adultos. Los machos y hembras fueron animales enteros y ninguna hembra se encontraba en gestación; criados en cautiverio bajo las mismas condiciones de manejo, alojados en 13 ambientes de 90 $\mathrm{m}^{2}$ cada uno, alimentados bajo una dieta con- 
sistente en forrajes, como kudzu (Pueraria phaseoloides), pasto elefante (Pennisetum purpureum), brachiaria (Brachiaria decumbens) y desmodium (Desmodium spp), y un concentrado a base de moyuelo y torta de soya.

El estado de salud de estos animales se determinó a través de la evaluación de constantes fisiológicas: temperatura (T), frecuencia cardiaca (FC) y frecuencia respiratoria (FR) medidas por los métodos convencionales y la observación del comportamiento (no variación de actitud, costumbres, etc.), consistencia y color de heces, orina, ausencia de secreciones nasales y cualquier alteración de las vías respiratorias, apetito, peso, estado de pelaje, ausencia de lesiones, traumatismo y parásitos en general.

\section{Obtención de muestras}

Para facilitar el adecuado manejo para la extracción de sangre, los animales fueron anestesiados mediante una combinación de xilazina y ketamina, en dosis de $0.25-0.5 \mathrm{mg} /$ $\mathrm{kg}$ pv y de $7-10 \mathrm{mg} / \mathrm{kg} \mathrm{pv}$ respectivamente, cargados en dardos de $3 \mathrm{cc}$ que se aplicaron vía intramuscular en la parte lateral de la región dorsal del cuello, en animales adultos, y en la región crural en animales juveniles.

La toma de muestras se efectuó entre las 6:00 y 9:00 horas. Tres ml. de sangre fueron extraídos por punción de la vena femoral, utilizando agujas calibre $\mathrm{N}^{\circ} 20 \mathrm{G} \mathrm{x}$ $1 \frac{1}{2}$, y colectadas en tubos estériles conteniendo el anticoagulante EDTA (etilenodiaminotetraacético) en la proporción de un $\mathrm{mg} / \mathrm{ml}$.

\section{Determinación de Valores Hematológicos}

Las muestras, fueron trasladadas al laboratorio del Centro de Reproducción y Conservación de Primates - Estación Experimental IVITA - Iquitos para determinar los valores hematológicos según la metodología descrita por Bush (1982):
Para la determinación del número de hematíes y leucocitos por $\mathrm{ml}$, se utilizó la cámara cuenta glóbulos de Neubauer y la pipeta de dilución de Thoma; usando como dilutores el ClNa al $0.85 \%$ para recuento de glóbulos rojos y la solución de $\mathrm{HCl}$ al $0.1 \mathrm{~N}$ para el recuento de leucocitos.

La determinación del hematocrito se efectuó utilizando capilares de $1 \mathrm{~mm}$ de diámetro por $75 \mathrm{~mm}$ de largo con EDTA, que contenían la sangre del ronsoco y fueron llevados a la microcentrífuga de alta velocidad (11,000 rpm por 5 minutos). La lectura en porcentaje se realizó directamente en una escala graduada, específica para dicho fin.

El recuento leucocitario se determinó en extensiones de sangre coloreadas con el colorante Wright; contándose 100 células, siguiendo la técnica de zig-zag de Shilling y los resultados se expresaron en porcentaje.

Para la determinación de hemoglobina se utilizó el método de la Oxihemoglobina, empleando el hemoglobinómetro de Spencer.

Los índices hematemétricos se calcularon utilizando los resultados de las determinaciones anteriores y aplicando las fórmulas respectivas. Así, se determinan el volumen globular medio (VGM) en fentolitros, hemoglobina globular media (HGM) en picogramos y concentración de hemoglobina globular Media (CHGM) en g/dl.

Los resultados obtenidos fueron sometidos a análisis estadísticos, utilizando la prueba de T de Student, para comparar los valores hallados en machos y hembras.

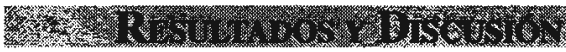

En los Cuadros 1 y 2 se presentan los valores hematológicos obtenidos en los 35 ronsocos, entre juveniles y adultos, clínicamente normales, en cautiverio en la 
Amazonía Peruana. Así mismo, en los Cuadros 3 y 4 se estos valores con relación al sexo.

En el presente estudio, la media en el número de eritrocitos fue de $3.19 \times 10 \% \mu l$ (3.08 $\times 10^{6} / \mu$ l para machos y $3.33 \times 10^{6} / \mu \mathrm{l}$ para hembras), valor ligeramente inferior a lo reportado por el International Species Information System, 1997 (ISIS) con una media de $3.50 \times 10^{6} / \mu \mathrm{l}$, para ronsocos sanos de zoológicos. Sin embargo, estos valores difieren de los reportados por Colveé (1976), quien reporta $4.67 \times 10^{6} / \mu \mathrm{l}$ para machos y $4.80 \times 10^{6} / \mu 1$ para hembras; que podrían explicarse por la ejecución de la técnica empleada, considerando que en el recuento de eritrocitos puede producirse un error estándar que alcanza $+15 \%$ (Schalm, 1983), así como a factores ambientales (altitud, clima, etc.), tipo de crianza (intensiva y extensiva) y alimentación.

El promedio de hemoglobina encontrado fue $14.32 \mathrm{~g} / 100 \mathrm{ml}(14.07 \mathrm{~g} / 100 \mathrm{ml}$ para machos y $14.62 \mathrm{~g} / 100 \mathrm{ml}$ para hembras), el cual coincide con los reportados por el ISIS cuya media es de $14.40 \mathrm{~g} / 100 \mathrm{ml}$, siendo superior a los hallados por Colveé (1976), quien reporta valores de $12.75 \mathrm{~g} / 100 \mathrm{ml}$ para machos y $13.13 \mathrm{~g} / 100 \mathrm{ml}$ para hembras. Esta diferencia puede deberse al tipo de alimentación o a la técnia utilizada ya que para este propósito existen varias técnicas como la Hematina Ácida, el método de la Oxihemoglobina y el de la Cianometahemoglobina con un error estándar de $15 \%, 10 \%$ y 1 a $2 \%$, respectivamente (Bush, 1982; Maximine, 1991 y Medway, 1973), desafortunadamente no se indican las técnicas utilizadas en ambos estudios.

En cuanto al hematocrito el valor promedio fue $42.23 \%$ (41.89\% para machos y $42.63 \%$ para hembras), y no presentan una diferencia marcada con los datos reportados por el ISIS cuya media es de $43.5 \%$, ni con los de Colveé (1976) que son de $43.09 \%$ y 46.92\% para machos y hembras, respectivamente.
Con relación a los índices hematemétricos, los resultados obtenidos para V.G.M., H.G.M. y C.H.G.M. fueron: 133.53 fl., 45.57 pg. y $34.42 \mathrm{~g} / \mathrm{dl}$, respectivamente (V.G.M. = 136.49 fl., H.G.M. $=46.38$ pg. y C.H.G.M. = 33.94 para machos y V.G.M. = 130.02 fl., H.G.M. $=55.50$ pg. y C.H.G.M.= 34.99 para hembras), mientras que llos valores hallados por Colveé (1976), fueron: V.G.M. $=136.49$ fl., H.G.M. $=46.38$ pg. $y$ C.H.G.M. = 33.94 para machos y V.G.M. = 130.02 fl., H.G.M. $=55.50$ pg. y C.H.G.M.= 34.99 para hembras y los reportados por el International Species Information System (1997) fueron en V.G.M. = 122.8 fl., H.G.M. $=41.3$ pg. y C.H.G.M. $=33.5$ g/dl. Las diferencias existentes entre el presente estudio y las otras investigaciones podrían ser consecuencia de los valores hallados en el número de eritrocitos, concentración de hemoglobina y porcentaje del hematocrito, debido a que las constantes hematemétricas siguen el mismo desplazamiento estadístico.

Respecto al número de leucocitos totales, el valor promedio obtenido fue de 5.40 $\mathrm{x} 10^{3} / \mu \mathrm{l}\left(5.21 \times 10^{3} / \mu \mathrm{l}\right.$ y $5.63 \times 10^{3} / \mu \mathrm{l}$ para machos y hembras, respectivamente). Estos valores son inferiores a los hallados por Colveé (1976), quien da a conocer $8.91 \times 10^{3}$ $/ \mu \mathrm{l}$ para machos y $8,64 \times 10^{3} / \mu \mathrm{l}$ para hembras, y a los reportados por el ISIS de 8.14 $\mathrm{x} 10^{3} / \mu$ l. Esto puede deberse a que el conteo total de leucocitos es significativamente influenciado por el lugar del drenaje de la sangre, la edad del animal y la actividad muscular (Maximine, 1991 y Schalm, 1983), que no se precisan en los trabajos antes indicados.

Para el caso de los neutrófilos de tipo abastonado, el valor promedio es de $0.94 \%$ (0.89\% para machos y $1.00 \%$ para hembras), coincidiendo con los reportados por el ISIS con un valor promedio de $1.09 \%$ aunque Colveé (1976) encontró valores de $1.45 \%$ en machos y $1.46 \%$ en hembras que son mayores a nuestros resultados. 
Cuadro 1. Valores promedio de la serie eritrocítica en 35 ronsocos (Hydrochaeris hydrochaeris), criados en cautiverio en la Amazonía Peruana.

\begin{tabular}{l|c|c}
\hline Variables & Media \pm I.C. & Rango \\
\hline Eritrocitos $\left(\times 10^{6} / \mathrm{ul}\right)$ & $3.19 \pm 0.157$ & $2.53-4.63$ \\
\hline Hemoglobina $(\mathrm{g} / \mathrm{dl})$ & $14.32 \pm 0.16$ & $12.5-16$ \\
\hline Hematocrito $(\%)$ & $42.23 \pm 1.52$ & $40-53$ \\
\hline VGM (fl $)^{1}$ & $133.53 \pm 5.92$ & $86.39-190.65$ \\
\hline HGM (pg) ${ }^{2}$ & $45.57 \pm 2.28$ & $31.32-58.46$ \\
\hline CHGM (g/dl) & $34.42 \pm 2.20$ & $25.96-42.86$ \\
\hline
\end{tabular}

1 Volumen globular medio.

2 Hemoglobina globular media.

3 Concentración de hemoglobina globular media.

Cuadro 2. Valores promedio de la serie leucocítica en 35 ronsocos (Hydrochaeris hydrochaeris), criados en cautiverio en la Amazonía Peruana.

\begin{tabular}{l|c|c}
\hline \multicolumn{1}{c|}{ Variables } & Media \pm I.C. & Rango \\
\hline Leucocitos (x 10\%lul) & $5.40 \pm 0.31$ & $3.55-7.80$ \\
\hline Mielocitos (\%) & 00 & 00 \\
\hline Metamielocitos (\%) & 00 & 00 \\
\hline Abastonados (\%) & $0.94 \pm 0.31$ & $0-4$ \\
\hline Segmentados (\%) & $51.89 \pm 3.06$ & $34-68$ \\
\hline Eosinófilos (\%) & $1.57 \pm 0.59$ & $0-9$ \\
\hline Basófilos (\%) & 00 & 00 \\
\hline Linfocitos (\%) & $42.26 \pm 3.21$ & $27-64$ \\
\hline Monocitos (\%) & 00 & 00 \\
\hline
\end{tabular}


Cuadro 3. Valores promedio de la serie eritrocítica en ronsocos (Hydrochaeris hydrochaeris), de acuerdo al sexo ( 16 hembras y 19 machos) criados en cautiverio en la Amazonía Peruana.

\begin{tabular}{l|c|c|c}
\hline \multicolumn{1}{c|}{ Variables } & Sexo & Media \pm I.C. & Rango \\
\hline \multirow{2}{*}{$\begin{array}{l}\text { Eritrocito } \\
(\mathrm{x} \mathrm{10} / \mathrm{ul})\end{array}$} & $\mathrm{M}^{*}$ & $3.08 \pm 0.23$ & $2.53-4.63$ \\
\cline { 2 - 4 } $\begin{array}{l}\text { Hemoglobina } \\
\text { (g/dl) }\end{array}$ & $\mathrm{H}$ & $3.33 \pm 0.23$ & $2.54-4.06$ \\
\cline { 2 - 4 } Hematocrito (\%) & $\mathrm{H}$ & $14.07 \pm 0.49$ & $12.5-16$ \\
\hline \multirow{2}{*}{ VGM (fl) ${ }^{1}$} & $\mathrm{M}$ & $41.89 \pm 2.66$ & $13-15.5$ \\
\cline { 2 - 4 } & $\mathrm{H}$ & $42.63 \pm 1.64$ & $34-53$ \\
\hline \multirow{2}{*}{ HGM (pg) } & $\mathrm{M}$ & $136.49 \pm 9.76$ & $86.39-190.65$ \\
\cline { 2 - 4 } & $\mathrm{H}$ & $130.02 \pm 7.64$ & $106.33-170.42$ \\
\hline CHGM (g/dl) & $\mathrm{M}$ & $46.38 \pm 3.58$ & $31.32-58.46$ \\
\cline { 2 - 4 } & $\mathrm{H}$ & $55.50 \pm 3.31$ & $35.44-56.65$ \\
\hline
\end{tabular}

$(*)$ No se encontraron diferencias significativas $(p>0.05)$ entre variables, para ambos sexos.

1 Volumen globular medio, 2 Hemoglobina globular media, 3 concentración de hemoglobina globular media

En cuanto a las células de tipo segmentado el valor promedio fue de $51.89 \%$ (50.05 para machos y $54.06 \%$ para hembras) el cual coincide con los reportados por el ISIS con un promedio de $55.42 \%$, pero difiere de los de Colveé (1976) de $12.10 \%$ y $10.31 \%$ para machos y hembras, respectivamente. Debido a que en el trabajo reportado por Colveé no se consignan datos sobre las condiciones de los animales objeto de su estudio no se puedé discutir la indicada diferencia.

El promedio de linfocitos encontrados en el presente estudio de $42.26 \%$ (47.63\% y
44.63\% para machos y hembras, respectivamente) es superior al reportado por el ISIS de $36.85 \%$; sin embargo es inferior al de Colveé (1976) de $63.45 \%$ en machos y $66.25 \%$ en hembras.

En el presente trabajo no se encontraron monocitos; contrariamente a lo reportado por el ISIS que reporta un valor promedio de $4.41 \%$, asímismo Colveé (1976) encontró $0.91 \%$ para machos y $1.54 \%$ para hembras.

Como en el caso anterior no se encontraron basófilos, lo que concuerda con 
Cuadro 4. Valores promedio de la serie leucocítica en ronsocos (Hydrochaeris hydrochaeris) de acuerdo al sexo (19 machos y 16 hembras), criados en cautiverio en la Amazonía Peruana.

\begin{tabular}{|c|c|c|c|}
\hline Variables & Sexo & Media \pm I.C. & Rango \\
\hline \multirow{2}{*}{$\begin{array}{l}\text { Leucocitos } \\
\left(\mathrm{x} 10^{3} / \mathrm{ul}\right)\end{array}$} & $\mathbf{M}^{*}$ & $5.21 \pm 0.41$ & $3.55-6.85$ \\
\hline & $\mathrm{H}$ & $5.63 \pm 0.50$ & $4.10-7.80$ \\
\hline \multirow{2}{*}{ Mielocitos } & $\mathbf{M}$ & 00 & 00 \\
\hline & $\mathrm{H}$ & 00 & 00 \\
\hline \multirow{2}{*}{ Metamielocitos } & $\mathbf{M}$ & 00 & 00 \\
\hline & $\mathrm{H}$ & 00 & 00 \\
\hline \multirow{2}{*}{ Abastonados } & $\mathbf{M}$ & $0.89 \pm 0.55$ & $0-3$ \\
\hline & $\mathrm{H}$ & $1.00 \pm 0.34$ & $0-4$ \\
\hline \multirow{2}{*}{ Segmentados } & $\mathbf{M}$ & $50.05 \pm 4.52$ & $34-63$ \\
\hline & $\mathrm{H}$ & $54.06 \pm 4.64$ & $35-68$ \\
\hline \multirow{2}{*}{ Eosinófilos (\%) } & $\mathbf{M}$ & $1.32 \pm 0.67$ & $0-6$ \\
\hline & $\mathrm{H}$ & $1.87 \pm 1.08$ & $0-9$ \\
\hline \multirow{2}{*}{ Basófilos (\%) } & $\mathbf{M}$ & 00 & 00 \\
\hline & $\mathrm{H}$ & 00 & 00 \\
\hline \multirow{2}{*}{ Linfocitos(\%) } & $\mathbf{M}$ & $47.63 \pm 5.05$ & $27-62$ \\
\hline & $\mathrm{H}$ & $44.63 \pm 4.58$ & $35-61$ \\
\hline \multirow{2}{*}{ Monocitos (\%) } & $\mathbf{M}$ & 00 & 00 \\
\hline & $\mathrm{H}$ & $\infty$ & 00 \\
\hline
\end{tabular}

(*) No se encontraron diferencias significativas $(p>0.05)$ entre variables, para ambos sexos. 
Colveé (1976), quien tampoco reporta la presencia de estas células; sin embargo el ISIS encuentra un valor promedio de $0.63 \%$.

El valor promedio de eosinófilos de $1.57 \%(1.32 \%$ y $1.87 \%$ para hembras y machos, respectivamente), es inferior al reportado por ISIS cuyo promedio es de $9.15 \%$. Colveé (1976) reporta valores promedio de $21.82 \%$ para machos y $20.23 \%$ para hembras; diferencia que no es posible explicar, aunque podría sugerirse un posible proceso parasitario o alérgico (Maximine, 1991).

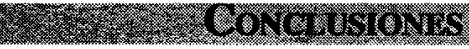

Los resultados que se indican en el presente trabajo constituyen el primer aporte relacionado con la hematología del ronsoco (Hydrochaeris hydrochaeris) en cautiverio en la Amazonía Peruana. No se encontraron diferencias significativas entre las variables correspondientes a machos y hembras.

La discusión comparativa con los valores obtenidos por otros autores es relativa por cuanto éstos, además de haberse realizado en diferentes ecosistemas, no aluden las técnicas utilizadas en la determinación de las variables, ni las características y/o sistemas de crianza de los animales estudiados.

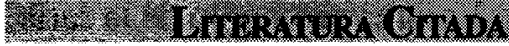

1. Bush, B. M. 1982. Manual de laboratorio veterinario de análisis clínicos. 1a. ed., p. 467. Editorial Acribia, Zaragoza, España.

2. Colveé, P. 1976. Parámetros sanguíneos en Chigüires (Hidrochaeris hidrochaeris) p. 116. Resumen en II Seminario sobre Chiguiires y Babas. CONICIT I.P.A. Fac. Agro. U.C.V. Maracay.

3. FAO, 1988. El capibara y su importancia para el desarrollo rural. Acta Cient. Venezolana. Vol. 45. Supl 2. p.25.

4. Guerci, A. 1985. Métodos de análisis clínicos y su interpretación.1a. ed., p. 513. Editorial El Ateneo. Buenos Aires, Argentina.

5. International Species Identification System. 1997. Clinical Pathology Records Report - ISIS / In House Reference Values Mammals. http:/ www.worldzoo.org.

6. Maximine, M. 1991. Manual de Patología Clínica en veterinaria. la. ed., p. 389. Editorial Limusa. México.

7. Medway, D. 1973. Patología Clínica Veterinaria. 1a. ed., p. 208-253. Editorial Centro Regional de ayuda técnica. México.

8. Ojasti J. 1972. El Chigüire. Rev. Defensa de la naturaleza. Caracas, 1: 4-14.

9. Schalm, O. 1983. Veterinary hematology. 3a. ed., p. 219-258. Editorial Filadelfia. 